\title{
Retour sur « Quand le rite devient technique »
}

\section{Pierre Bonte}

\section{OpenEdition}

Journals

Édition électronique

URL : https://journals.openedition.org/tc/5089

DOI : $10.4000 /$ tc. 5089

ISSN : 1952-420X

\section{Éditeur}

Éditions de l'EHESS

\section{Édition imprimée}

Date de publication : 30 juin 2010

Pagination : $544-546$

ISSN : 0248-6016

\section{Référence électronique}

Pierre Bonte, «Retour sur «Quand le rite devient technique »», Techniques \& Culture [En ligne], 54-55 |

2010, mis en ligne le 30 juin 2013, consulté le 29 septembre 2022. URL : http://

journals.openedition.org/tc/5089; DOI : https://doi.org/10.4000/tc.5089 


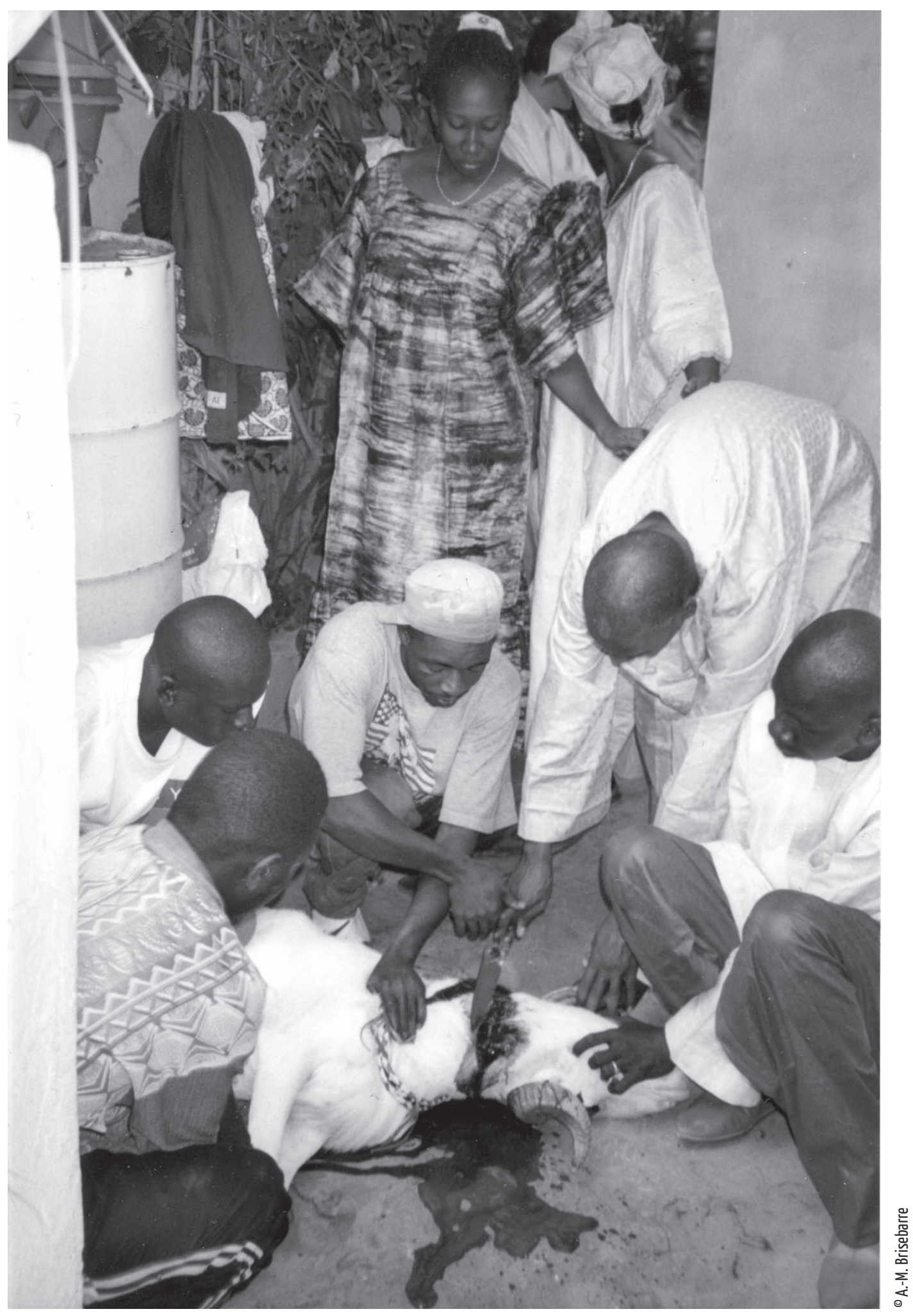




\section{Retour sur}

\section{«Quand le rite devient technique »}

Le texte qui suit répondait à une demande particulière - réexaminer la notion de «tendance » qu'André Leroi-Gourhan avait placée au point de départ de son analyse des techniques - qui en explique la démarche rhétorique privilégiant le rapport entre tendance et matière en fonction des modes d'action des techniques sur celle-ci. Leroi-Gourhan illustre l'ancrage des techniques humaines dans des lois physico-chimiques (percussion, propulsion, etc.) mettant en forme sociale les chaînes opératoires. Qu'en est-il cependant quand il s'agit de la matière vivante, en l'occurrence du rapport entre l'homme et l'animal sur lequel je me suis plus particulièrement penché? La mise à mort d'un animal en vue de la consommation de ses chairs est bien un fait technique, mais c'est aussi, assez universellement me semble-t-il, un fait rituel qui, sous sa forme sacrificielle, conjugue indistinctement efficacité technique et efficacité symbolique, si bien qu’adopter par exemple une alimentation végétarienne implique qu'on s'interdise le recours à la technique!

Dans les communautés musulmanes la production de viande halal est un fait technique qui se déroule selon une chaîne opératoire précise, mais ce fait opère aussi bien en ces circonstances qu'à l'occasion des gestes rituels effectués dans les temps et lieux des sacrifices. Le développement des consommations de masse et la «marchandisation» des produits, justifie le constat établi dès le titre: le rite devient de plus en plus « technicisé », intégré à la production industrielle de viande dans les abattoirs. L'étiquette halâl (licite), appliquée à de multiples autres produits de consommation devient quant à elle l'icône identitaire de l'appartenance à l'islam à l'époque contemporaine.

Les recherches menées depuis la rédaction de cet article m’ont permis de revenir sur certains thèmes à peine effleurés alors. Je suis ainsi de plus en plus convaincu de l'intérêt et de l'importance de conjuguer l'approche des techniques et celle des ethnosciences encore balbutiante à l'époque où Leroi-Gourhan composait ses œuvres majeures. La mise à mort 
des animaux s'inscrit dans des règles de classification précises qui distinguent les animaux licites et illicites, mais concernent aussi les substances corporelles que partagent ces animaux avec l'homme: le sang doit être séparé de la carcasse dès la mise à mort dont son écoulement brutal est la cause exclusive, sous peine de disposer d'une « charogne » (mayla) inconsommable. La licéité ou l'illicéité du produit est ainsi définie selon des règles d'organisation de la matière vivante qui donnent sens à l'acte technique et l'organisent. Ces règles opèrent dans le cadre de systèmes symboliques qui inscrivent l'homme dans cette matière vivante et prolongent la métaphore et la métonymie de manière analogique à partir du corps humain: traitement des substances (sang, lait, sperme, etc.), de la fécondité et des fonctions vitales, de la mort, etc.

Peut-on enfin rapprocher André Leroi-Gourhan et Claude Lévi-Strauss? La domestication, transformation radicale des rapports entre les sociétés humaines et leur environnement, ici la matière vivante, nous y incite en soulignant que l'animal est « bon à penser ». Les Nuer, soulignait E.E. Evans Pritchard élèvent leur bétail, outre la production de lait, pour produire du lien social: se marier et activer leurs relations de solidarités lignagères à l'occasion des rites sacrificiels, l'idée de tuer un animal pour s'alimenter est rejetée par eux avec dégoût. Chez les Grecs anciens se confondaient les personnages du boucher et du sacrificateur. La différence que l'on opère parfois, dans le monde musulman, entre abattage rituel et sacrifice, technique et rite, apparaît secondaire dans cette perspective. Les sacrifices canoniques (sunna), effectués lors du pèlerinage et dans l'ensemble de la 'umma en commémoration du sacrifice demandé par Dieu de son fils à Ibrahîm/Abraham, ainsi que pour la naissance d'un enfant s'inscrivent dans les représentations accompagnant la naissance d'un premier né et renvoient à une question d'ordre métaphysique: comment du féminin, une femme, peut naître le masculin, le premier garçon, rapprochant deux principes radicalement distincts dont le sacrifice nous offre la lecture inscrite dans le corps signifiant des victimes ainsi producteur des sens qu'offrent en excès la mise à mort et la découpe.

Dans les sociétés que Louis Dumont définit comme holistes, cette lecture est en effet ouverte. La distinction classificatoire canonique musulmane entre licite et illicite introduit un ordre qui découle de la Loi Révélée. Elle ne détermine pas totalement un autre classement en terme de pur et impur qui s'articule avec elle. Le sang consacré à la Divinité est pur, support des âmes animale et humaine, traitées de même, et en même temps impur et dangereux. Des sacrifices transgressifs, dans le cadre de rites adorcistes, s'observent dans la plupart des sociétés musulmanes s'accompagnant de consommation et d'aspersion du sang.

La distinction entre efficacité technique et efficacité symbolique continue certes à présenter un intérêt analytique et perpétue la valeur heuristique de la technologie culturelle. Mais l'étude des techniques doit prendre garde à ne pas céder à la tentation positiviste alimentée par les représentations de l'individualisme moderne. L'évolution industrielle de la production de biens halâl et leur marchandisation constituent à l'inverse une perte de sens qui n'en retient que les signes identitaires. La «bio-technologisation » du vivant est source d'apories. Le manifestent aussi, par ailleurs, les conséquences du développement des techniques de procréation assistée dont l'effet imprévu est de tendre à réduire la riche notion sociale et symbolique de filiation à la transmission génétique et au rapport individuel avec les origines biologiques. 